\title{
Helping Relationships and Genetic Propensities: A Combinatoric Study of DRD2, Mentoring, and Educational Continuation
}

\author{
Michael J. Shanahan,' Lance D. Erickson, ${ }^{2}$ Stephen Vaisey,' and Andrew Smolen ${ }^{3}$ \\ ' University of North Carolina, Chapel Hill, United States of America \\ ${ }^{2}$ Brigham Young University, Utah, United States of America \\ ${ }^{3}$ University of Colorado, Boulder, United States of America
}

Erom rom conception to death, helping relationships promote positive development and enable people to surmount challenges in their lives. Is it the case that the negative consequences of a genetic propensity for risky behaviors can be attenuated by helping relationships ( $\mathrm{a} \times \mathrm{E}$ )? But is it also the case that people with such a genetic propensity are less likely to have helping relationships compared to people without such a propensity (a rGE)? We illustrate this complex pattern of gene-environment interplay by drawing on the National Longitudinal Study of Adolescent Health and a combinatoric analytic strategy. We focus on a gene associated with dopamine receptor type 2 (DRD2 TaqIA), student-mentor relationships, and educational continuation beyond secondary school. Results reveal that, for both white and black males, DRD2 A1+ (A1A1 and A1A2 genotypes) is associated with a decreased likelihood of school continuation compared to their counterparts with DRD2 A1-; mentors who are teachers compensate for this negative association ( $G \times E$ ); and youth with DRD2 A1+ are less likely to have a mentor who is a teacher than their counterparts with DRD2 A1- (a rGE).

Helping relationships - involving one's parents, peers, teachers, mentors, spouses, adult children, fellow congregants, neighbors, and so on - are typically thought to foster adjustment and health, broadly conceived, by promoting positive development and enabling people to surmount adversities. Yet what if people with a genetic propensity for risky behavior are more likely to benefit from helping relationships but less likely to have them when compared with people without such a propensity? This pattern suggests both a gene-environment interaction (i.e., those with a genetic propensity for risky behavior will benefit more from a helping relationship than those without such a propensity) and a gene-environment correlation (these same people will be less likely to have a helping relationship). This paper explores these complications, focusing on the case of the student-mentor relationship, DRD2, and continuation beyond secondary school, which is a strategic empirical example.

In the American context, educational continuation beyond secondary school has major implications for adulthood, including life-long patterns of income (Geweke \& Keane, 2000; Restuccia \& Urrutia, 2004) and physical and mental health (Avison, 2005; Dohrenwendet al., 1992; Miech \& Shanahan, 2000; Ross \& Wu, 1995; Schnittker, 2004). In turn, income and health are related to myriad aspects of successful aging - including subjective assessments of life satisfaction, marital quality, and community involvement - suggesting a pivotal and crucial role for education in the life course.

Mentoring is of special significance because models attempting to predict which students continue beyond secondary school have traditionally focused on the socioeconomic status of the parents and the educational aspirations that they maintain for their children (Sewell et al., 1969). But such a view has expanded to include influential, nonparental adults (mentors) and indeed research suggests that youth who are involved in a mentoring relationship experience more academic success than their peers without such relationships (Bryant \& Zimmerman, 2003; Hamilton \& Darling 1996; Klaw et al., 2003; Zimmerman et al., 2002). Nevertheless, most prior research focuses on 'at-risk youth' with little attention to comparatively diverse samples (Rhodes et al., 1994; Rhodes et al., 1992; Zimmerman et al., 2002).

We consider DRD2 Taq IA, a gene that is associated with dopamine type 2 receptors. DRD2 has been linked with phenotypes that would be disadvantageous in educational settings. Positron emission tomography shows that the presence of the DRD2 TaqI A1 allele is associated with lower density of

Received 6 December, 2006; accepted 31 January, 2007.

Address for correspondence: Michael Shanahan, Department of Sociology, University of North Carolina, Chapel Hill, CB 3210, Hamilton Hall, Chapel Hill, NC, 27599-3210, USA. E-mail: mjshan@unc.edu 
dopamine receptors and a reduced number of dopamine binding sites in the striatal region in the brains of healthy, living humans (Jonsson et al., 1999; Oohjalainen et al., 1998), perhaps more so in the ventral caudate and putamen (Thompson et al., 1997). These regions of the brain have been associated with the reward system, impulse control, planning, and related behaviors, and the dopaminergic system has been linked to reward-seeking behavior in humans (e.g., Pessiglione et al., 2006). Studies with nonhuman animals suggest a role of D2 and D2-like receptors in premature responding and impaired accuracy of responses (Pattij et al., 2006), impulsive choice making (van Gaalen, Brueggeman, et al., 2006), inhibitory control (van Gaalen, van Koten, et al., 2006), and deficits in the initial acquisition of task-governing rules (Glickstein et al., 2005). The Online Mendelian Inheritance in Man also suggests behavioral correlates including alcoholism, Parkinson disease, schizophrenia, and Tourette's syndrome (Online Mendelian Inheritance in Man, 2006). Taken as a whole, these and related studies (for example, using DRD2 knockout mice, see Kruzich et al., 2006; Pezze et al., 2007; Smith et al., 2002; Tran et al., 2002) suggest that although the exact associations among DRD2 alleles, specific neural processes, and specific phenotypes are not presently understood, DRD2 A1+ is associated with phenotypes that would undermine school performance and educational attainment.

The present study focuses on (1) whether DRD2 $\mathrm{A} 1+$ is associated with continuation to college/university; and, if so, (2) whether mentoring relationships can attenuate this association (i.e., a $\mathrm{G} \times \mathrm{E}$ ); and finally, (3) whether DRD2 A1+ is associated with having mentors (i.e., a rGE). In addition to these substantive interests, we illustrate the usefulness of a combinatoric approach to studying gene-environment correlations and interactions by applying a novel analytic method to the study of genes and behavior, Qualitative Comparative Analysis (QCA), and by drawing on molecular genetic data available in Wave III of the National Longitudinal Study of Adolescent Health (Add Health). In so doing, our paper highlights a new analytic strategy for researchers interested in the interplay of genes and environmental factors, a point to which we return in the Conclusions and Discussion.

\section{Mentoring: DRD2 Interplay and School Continuation}

Given the relationship between DRD2 and phenotypes that would be disadvantageous in classrooms, we expect that DRD2 A1+ will be associated with poor performance in social settings that call for high levels of self-control, such as schools, workplaces, and families. In the school setting, a career of such behaviors - possibly including substance abuse and impulsivity - will culminate in a lowered probability of matriculation to college/university (Hypothesis 1 ).
Could this relationship be buffered by mentoring? If so, do students with DRD2 risk develop mentoring relationships in the first place?

\section{Mentors and DRD2}

Mentoring represents a form of social capital, which refers to links among actors (in this case, the student and the mentor) that facilitate action (in this case, educational continuation; Portes, 1998). Prior research distinguishes between formal and informal mentoring and most studies of mentors focus on the former: youth who are candidates for organized mentoring programs due to a high risk of failure, at least in terms of their educational prospects (see Rhodes et al., 2000) or their social disadvantage (e.g., Bryant \& Zimmerman, 2003; Rhodes et al., 1994). Informal mentors are relatives, friends, and other community members with whom youth have developed relationships through their existing social networks.

The distinction between formal and informal mentors maps onto the distinction between bridging and bonding social capital. Bridging social capital connects actors who differ with respect to salient resources and thus bridging capital is believed to be especially important in reducing soecioeconomic inequalities (including education). Formal mentors are likely to represent bridging capital because programs assign people with special skills and knowledge to assist students without such skills and knowledge. Teachers who are mentors, although perhaps not formal mentors, also represent bridging capital because they possess a high level of experience with and insight into the educational system. In contrast, bonding social capital connects actors who do not differ appreciably in their salient resources. Accordingly, informal mentors who are not teachers are likely to lack expertise in the educational system and thus constitute bonding capital.

These distinctions suggest that teachers as mentors may be especially salient forms of bridging social capital. Because of their expertise with the educational system and personal knowledge of the student, a mentor who is a teacher may contribute to school continuation in the presence of DRD2 A1+ (Hypothesis 2) by encouraging appropriate behavioral comportment and good decisions about schooling. On the other hand, a nonteacher mentor, lacking these insights and skills, may be of little assistance (Hypothesis 3).

Are some youth more likely to have teachers as mentors? Rhodes (2002) suggests that adolescents who are competent and goal-driven may appear a 'better investment' of a teacher's time than youth who are impulsive and lack planful competence. Thus, youth with DRD2 A1+ are expected to attract fewer teachers as mentors than youth with A1- (Hypothesis 4). Taken as a whole, these hypotheses suggest that teachers may be especially beneficial to students with DRD2 A1+, but that these very same students are less likely to attract teachers as their mentors. 


\section{Differences by Sex and Race}

Could these processes be complicated by sex and/or race? Most of the evidence that suggests links among DRD2 alleles, neurobiology, and behavior is silent on issues of sex and race. Biological studies that link DRD2 with neurobiological structure and function restrict attention to males or often fail to find male-female differences. Thus, for example, DRD2 risk is presumed to lower neuronal density and binding site availability equally in the striatal regions of males and females, and whites and blacks. Likewise, the animal literature linking DRD2 allelic variation with behavior typically focuses on males (e.g., Wistar rats) and the race distinction obviously has no nonhuman animal homologue. Finally, studies linking DRD2 alleles and behaviors in humans do not provide consistent evidence for sex or race differences, with apparently no attention devoted to the latter.

Nevertheless, sex and race differences in the proposed hypotheses may be observed. With respect to sex, females and males are socialized differently, such that many behaviors that would be deemed inappropriate in the classroom (e.g., disruptive behaviors) would be considered especially problematic for females. Thus, the effect of DRD2 on continuation may be smaller for females than for males (Hypothesis 5).

Some theories of social capital maintain that it serves the majority's interest to reproduce inequalities (e.g., Bourdieu \& Passeron, 1990), an argument typically construed in terms of socioeconomic strata but applicable to race distinctions as well. Stanton-Salazar and Dornbusch (1995) argue that racial/ethnic minorities find supportive ties in school settings and community organizations, but these ties are often problematic because institutional agents of the school (e.g., teachers, coaches) tend not to share subcultural values and norms with nonwhites. This line of reasoning suggests that teachers may have little or negative association with continuation among blacks (Hypothesis 6). In effect, hypotheses 5 and 6 suggest that blacks, particularly black males, will not benefit from either bridging or bonding capital.

\section{Sample}

The National Longitudinal Study of Adolescent Health (Add Health) was based initially on a nationally representative sample of youth in grades 7 through 12 in the United States. Data have been obtained from the adolescents themselves, and from their parents, siblings, friends, romantic partners, and from school administrators. The National Quality Education Database provided the sampling frame with its list of all high schools in the United States $(N=26,666)$. To qualify, a high school had to include an 11th grade and an enrollment of more than 30 students. From this frame 80 schools were selected. The sample was stratified according to several distinctions, including region, suburban/urban/rural, school type (whether public, private, parochial), ethnic mix, and size. Fifty-two of the 80 schools agreed to participate, and 28 replacements schools were selected based on the stratifying variables. Each of the 80 schools was paired with a middle school (based on its contribution to the high school student body). A total of 145 of the schools agreed to host a confidential in-school survey which focused on adolescent health and friends. This first wave yielded 90,118 students from grades 7 to 12 (in 1994). Nearly four out of five schools provided a roster from which the first in-home interview sample was drawn.

From the rosters, students were randomly selected for a 90-minute interview, conducted in the home. Approximately 200 students were recruited from schools in each school pair, regardless of size. This procedure resulted in a self-weighting sample. A total of 20,745 adolescents in grades 7 through 12 (ages 11 through 19) were interviewed at home. This in-home wave of interviews with target child and parent was carried out in 1995, between April and December. The second in-home interview encompassed youth who were in the 7 th to 11 th grades at Wave I, was comparable to the first interview in its coverage, and was carried out between April and August of 1996. The Wave II in-home survey resulted in a sample of 14,738 youth between the ages of 13 and 20. A third wave was collected between August 2001, and April 2002 from Wave I participants, resulting in 15,197 18- to 26-year-olds.

The present study draws on data collected from students and parents at Wave I and from youth at Wave III. In Wave I, data were collected from a sibling pairs subsample; $64 \%$ of the cases arose from the Add Health probability sample, and $36 \%$ of the pairs were collected as a convenience sample. The sibship sample is composed of 1249 full-sibling pairs, 424 half-sibling pairs, and 657 biologically unrelated pairs. DNA data were collected from some of the sibling pairs at Wave III $(n=2574)$, with genotyped DRD2 data available for 2557 individuals. The average sibship size among the 2557 individuals is 1.88 , with a standard deviation of .46 ; an inspection of the frequency distribution for sibship size shows that almost $79 \%$ of the youth have one other sibling in the dataset and about $17 \%$ are singletons.

Thus, the DNA subsample of Add Health is not a probability sample. Comparisons between the fullyweighted Wave I school-based dataset and the DNA subsample (not shown; available upon request) reveal similar levels of parental socioeconomic status, parental involvement in school, and school quality, and somewhat higher levels of talking to parents about school in the latter. These comparisons also reveal that the DNA sample is more educated, more likely to belong to a nonintact family, more likely to live in the West (as opposed to Northeast), and more suburban (as opposed to rural).

Finally, among the 2557 individuals in the DNA subsample, there were missing data: about $10 \%$ for 
variables from the parent dataset, about $2 \%$ for the Wave I youth data, and less than $1 \%$ for the Wave III measure of educational continuation. All analyses are based on 10 imputed datasets, resulting in an efficiency of estimates exceeding 97\% (Rubin, 2004).

\section{Measures \\ DRD2}

The gene spans over $270 \mathrm{~kb}$ with a large first intron $(250 \mathrm{~kb})$ and maps to $11 \mathrm{q} 22.3-11 \mathrm{q} 23.1$ (Eubanks et al., 1992). The TaqIA polymorphism (Grandy et al., 1989 ) is the most commonly studied and is located about $10 \mathrm{~kb}$ down stream from exon 8 , which is the end of the DRD2 gene (Kidd et al., 1996). The TaqIA polymorphism refers to a single nucleotide polymorphism (SNP) in the 3' untranslated region of the gene (and so may act via an associated, functional polymorphism in an adjacent gene). The A1 allele has a point mutation $\mathrm{C} \rightarrow \mathrm{T}$ (TCGA to TTGA), which eliminates the TaqI site. This polymorphism was assayed at the Institute of Behavioral Genetics, University of Colorado-Boulder.

The DRD2 TaqIA assay (Haberstick \& Smolen, 2004) was performed using the fluorogenic 5 'nuclease (Taqman ${ }^{\circledR}$, Applied Biosystems, Foster City, CA). Unlabeled forward and reverse primers were purchased from Integrated DNA Technologies (Coralville, IA). VIC and FAM labeled probes were puchased from ABI. All reactions were performed in an ABI Prism ${ }^{\circledR} 7000$ Sequence Detection System using the allelic discrimination mode (Livak, 1999). Reactions contained $1 \mu \mathrm{l}$ (approximately $20 \mathrm{ng}$ ) of DNA, $8.5 \mu \mathrm{l}$ of water, $0.5 \mu \mathrm{l}$ of $40 \mathrm{x}$ primer/probe mix and $10 \mu \mathrm{l}$ of $2 \times$ Taqman ${ }^{\circledR}$ Universal Master Mix. Final primer and probe concentrations were $900 \mathrm{mM}$ and $200 \mathrm{mM}$, respectively. The seqences of primers and probes were: Forward Primer: 5'-GTGCAGCTCACTCCATCCT-3'; Reverse Primer: 5'GCAACACAGCCATCCTCAAAG-3'; Probe 1: 5'VIC-CCTGCCTTGACCAGC-NFQMGB-3'; Probe 2: 5'- FAM-CTGCCTCGACCAGC-NFQMGB-3'.

Probe 1 (VIC/NFQMGB) anneals to the 'A'-containing SNP (TaqIA-1, $304 \mathrm{bp}$, no restriction site) and probe 2 (FAM/NFQMGB) anneals to the ' $G$ 'containing SNP (TaqIA-2, $178 \mathrm{bp}$, containing the restriction site.) Cycling parameters used were a 10 minute hold at $95^{\circ} \mathrm{C}$ followed by 40 cycles of $92^{\circ} \mathrm{C}$ for 15 seconds and $60^{\circ} \mathrm{C}$ for 60 seconds. Each 96 well plate included six nontemplate controls, four samples homozygous for the A SNP, four samples homozygous for the G SNP and two heterozygous samples. Genotypes were scored independently by two individuals. Preliminary analyses supported the distinction between DRD2 A1+ (A1A1 and A1A2) and A1- (those students homozygote for A2), a distinction supported by many other studies (e.g., see Noble's 2003 review). Research conducted at the Institute of Behavioral Genetics indicates that DRD2 is in Hardy-Weinberg Equilibrium (Smolen, January 2007, personal communication).

\section{Mentors}

All mentoring data come from the Wave III in-home interview data and were reported by the young person. Informal mentors were identified with the following question: 'Some young people know adults, other than their parents, who make an important positive difference in their lives. Some do not. Has an adult, other than your parents or stepparents, made an important positive difference in your life at any time since you were 14 years old?'. If there was more than one influential adult, respondents were asked to report only on the most important. The mentor's social role, or relationship to the young person, was identified by the respondent as an adult relative (older brother, younger brother, older sister, younger sister, mother's mother, mother's father, father's mother, father's father, aunt, uncle, spouse or partner), friend, teacher (teacher or guidance counselor, coach/athletic director), or community member (minister, priest, rabbi, or religious leader, employer, co-worker, neighbor, friend, , friend's parent, doctor/therapist/social worker, other). These two items - whether a respondent had a mentor and, if so, the role of the mentor - were used to create three sets: no mentor, mentor who was not a teacher, and mentor who was a teacher. Finally, the youth were asked 'How old were you when \{HE/SHE $\}$ first became important in your life?'. This item, combined with the mentor status item, were used to assure that mentors became important after age 14 but before age 18 (i.e., during high school for the vast majority of youth).

\section{Parental Socioeconomic Status}

Research on educational continuation traditionally focused on the important role of parental socioeconomic status and so we include this variable in our analysis. Socioeconomic status is the average of two variables standardized (mean $=0$ and unit variance), highest completed parental education $(1=8$ th grade or less, $2=$ more than 8 th grade but did not complete high school, 3 = went to business, trade, or vocational school instead of high school, $4=$ high school graduate, 5 = completed a Graduate Equivalency Diploma, $6=$ went to business, trade, or vocational school after high school, 7 = went to college but did not graduate, 8 $=$ graduated from college or university, $9=$ professional training beyond a 4 year college or university) and logged household income (ranges from $\$ 0$ to $\$ 999,000$ ).

\section{Physical Appearance and Attractiveness of Personality.}

Although the analyses focus on DRD2 and mentors, other factors undoubtedly contribute to the elicitation of help from mentors. We include two such factors, physical attractiveness and attractiveness of personality. After the interviewer finished administering the Wave I In-Home materials, he or she answered the following questions on a 5-point Likert scale: 'How physically attractive is the respondent?' and 'How attractive is the personality of the respondent?'. 


\section{School Continuation}

Respondents were asked 'What is the highest grade or regular year of school that you have completed?'. This items was then used in conjunction with six other items asked about degrees received to categorize youth into two sets: did not continue education beyond high school; continued education beyond high school.

\section{Analytic Strategy}

The hypotheses focus on whether DRD2 alleles are related to school continuation (Hypothesis 1); teachers as mentors can attenuate the association between DRD2 and school continuation (Hypothesis 2); nonteacher mentors will not attenuate this association (Hypothesis 3); and students with DRD2 risk are less likely to attract teachers as mentors then students without such risk (Hypothesis 4). Further, we expect that the link between DRD2 risk and continuation rates will be stronger for males than for females (Hypothesis 5) and that the positive effect of teachers will not be observed among blacks (Hypothesis 6).

Hypotheses 1 and 5 are addressed with a simple comparison of continuation rates across groups defined by DRD2's alleles. The remaining hypotheses are addressed with QCA (Ragin, 1989). QCA is an analytic method that combines Boolean algebra with descriptive statistical analysis. A 'set' refers to a group of people defined by one independent variable. Combinations of sets are called 'configurations.' Rather than trying to discover the net causal effect of single independent variables, QCA assesses how each configuration compares to other configurations on their level of the dependent variable. In so doing, QCA is well-suited to the study of gene-environment interplay.

Consider a simplified example: a theory predicting that $\mathrm{Y}$ reflects $\mathrm{A}$ (a gene), B (a contextual variable), and $C$ (another contextual variable), all of which are dichotomous variables (i.e., 0 or 1 ). A, B, and $\mathrm{C}$ each define a set. Rather than estimating the net effect of each of A, B, and C, QCA instead creates an 8-cell typology that combines their possible values (i.e., three variables with two response categories $=2^{3}=8$ configurations). We can then examine how many people are in each configuration compared with expectations given the marginals, which could reveal a $\mathrm{r}(\mathrm{GE})$. We can also compare the proportion of people with $Y=1$ across the eight configurations, which would reveal a $\mathrm{G} \times \mathrm{E}$. If we found, for instance, that both $\mathrm{A} \cdot \mathrm{b} \cdot \mathrm{C}^{1}$ and $\mathrm{A} \cdot \mathrm{B} \cdot \mathrm{C}$ stood out positively relative to the other configurations, we could use Boolean algebra to reduce this solution to A.C. (B and b cancel each other out as long as they are the only differences between the solutions.) That is, a specific polymorphism (A) accompanied by a specific context (C) significantly increase the likelihood that $\mathrm{Y}=1$ and $\mathrm{B}$ is irrelevant.

There are several advantages to QCA compared with more traditional methods. First, it takes seriously the fact that real life may reflect high levels of contigencies among predictor variables. Second, it provides a model that treats 'interactions' between variables with greater sensitivity than general linear models. In a general linear framework, for example, the interaction term $(\mathrm{A}) \times(\mathrm{B})$ would be scored 0 if the respondent was either a or $\mathrm{b}$ or both. It therefore treats potentially very different kinds of situations as the same type of thing. QCA preserves these differences by looking at all configurations.

In the present analyses, we are interested in differences in school continuation rates. To form the explanatory configurations, we rely on six variables: DRD2 A1+ $(G)$, having a mentor $(M)$, having a teacher as a mentor (T), high parental socioeconomic status (S), physically attractive (A) and attractive personality $(\mathrm{P})$.

The 'crisp variant' of QCA (explained above) assumes that each person is (1) or is not (0) a member of each configuration of sets. A crisp set approach thus yields 48 configurations of sets and each person is a member of only one of these configurations. (There would be 26 (or 64) configurations except that $\mathrm{m}^{* \mathrm{~T}}$ is logically impossible.) For example, a person in the configuration of sets 'youth with no DRD2 risk, has a mentor, that mentor is not a teacher, high parental status, not physically attractive, and attractive personality' would belong to that configuration of sets (i.e., their membership would equal 1 for a dichomotomous variable representing that configuration of sets) and would not belong to any of the other configurations of sets (i.e., membership $=0$ for each of the other 47 configuration of sets).

Given low subsample sizes, particularly for the black males and females, we employ a fuzzy set variant of QCA, which allows for partial membership in sets. Accordingly S, A, and P are 'fuzzified' by converting their $z$ scores into their equivalent place on the cumulative normal distribution, which has a natural range of 0 to 1 , noninclusive. (The DRD2 variable $G$ and the mentoring variables $M$ and $T$ necessarily remain dichotomous.) In order to provide sample-specific fuzzy membership, we linearly rescale these values such that the minimum for each set is 0 and the maximum is 1 . Following standard practice (Ragin, 2000), we combine individual sets into configurations using the minimum value of the constituent sets. For example, a respondent with $\mathrm{G}=1, \mathrm{M}=1, \mathrm{~T}=1$, $\mathrm{I}=.44, \mathrm{~S}=.90$, and $\mathrm{A}=.85$ would have membership .44 in the set $\mathrm{G} \bullet \mathrm{T} \bullet \mathrm{S} \bullet \mathrm{I} \bullet \mathrm{Q} \bullet \mathrm{A}, \quad 0$ in the set $\mathrm{g} \bullet \mathrm{T} \bullet \mathrm{S} \bullet \mathrm{I} \bullet \mathrm{Q} \bullet \mathrm{A}$, and .10 in the set $\mathrm{G} \bullet \mathrm{T} \bullet \mathrm{s} \bullet \mathrm{I} \bullet \mathrm{Q} \bullet \mathrm{A}$. That is, most respondents will provide information about a number of configurations of sets simultaneously.

Drawing on fuzzified sets and their configurations, we consider the broad issue of $\mathrm{G} \times$ Es involving DRD2 and types of mentors using two strategies. First, for each one of the 48 configurations, we statistically compare its school continuation rate with the average rate of the 47 other groups. To allow for such comparisons, we generate values for 'proportion 
continuing' by using the formula for fuzzy inclusion: Isk,y $=\Sigma \min (\mathrm{sk}, \mathrm{y}) / \Sigma$ sk, where Isk,y is the extent to which set $s$ is included in outcome set $y$, and $k$ indexes which of the 48 configurations is under consideration. ${ }^{2}$ Each configuration of sets is then compared to the average of the other 47 using an $F$ test. (The formula automatically weights the responses so that respondents with low values of a configuration count less toward producing the value of Isk,y than do respondents with high values of a configuration.) Translating this into set-theoretic language, this formula essentially asks the question 'to what extent is this configuration $(\mathrm{X})$ a subset of the outcome (Y)?'. The resulting value (of Isk,y) can range from 0 ( $\mathrm{X}$ and $\mathrm{Y}$ do not overlap at all) to 1 ( $\mathrm{X}$ is completely included in $\mathrm{Y}$ ).

These comparisons identify configurations that are significantly different from the rest of the population in their rates of continuation (either positively or negatively). Among configurations with a significantly higher continuation rate (i.e., 'positive stand-out configurations'), we then use Boolean algebra to simplify the solution (e.g., A.B.C plus $\mathrm{A} \cdot \mathrm{b} \cdot \mathrm{C}$ reduces to $\mathrm{A} \cdot \mathrm{C}$ ). The same procedure is also applied to the negative stand-outs, or those configurations with significantly lower continuation rates. These comparisons thus identify configurations of DRD2 and mentorships that are sufficient to produce results that are significantly different from other configurations that are closer to the overall sample's continuation rate.

Second, we focus on genetic differences in continuation rates within social capital configurations. Combining the variables (M, T, S, A, and P) yields 24 possible configurations. Within each of these 24 configurations, do continuation rates differ by genetic variation (i.e., DRD2 $\mathrm{A} 1+$ and $\mathrm{A} 1-$ )? We address this question by conducting an $F$ test of the difference in school continuation rate between these two groups. These analyses compare continuation rates between DRD2 A1+ and A1- groups in the same configuration of sets of social context.

All analyses were conducted with Stata and fsQCA software packages and adjust standard errors for nonindependence of observations due to sibships.

Table 1

Continuation to Tertiary Level by Race, Sex, and DRD2 Allele, $\%$ and $n$

\begin{tabular}{lcccc}
\hline & \multicolumn{2}{c}{ White } & \multicolumn{2}{c}{ Black } \\
\hline & Male & Female & Male & Female \\
\hline DRD2 A1+ & $44.4,360$ & $57.6,363$ & $34.7,121$ & $47.1,153$ \\
DRD2 A1- & $59.3,513$ & $61.4,581$ & $51.5,99$ & $51.8,114$ \\
\hline$z$ statistic, $p$ & $3.90, .000$ & $1.07, .283$ & $2.54, .011$ & $.68, .493$ \\
$\begin{array}{l}\text { Note: Logistic regression is used to compute significance tests to adjust the } p \text { values } \\
\text { for using 10 imputed datasets. A1+ includes A1A1 and A1A2 genotypes; A1- } \\
\text { includes the A2A2 genotype }\end{array}$
\end{tabular}

\section{$\overline{\text { Findings }}$}

Is DRD2 associated with continuation to the tertiary level? Table 1 reports the mean continuation rates for groups defined by race, sex, and DRD2. For males, continuation rates are significantly different between DRD2 A1+ and A1- groups, and the differences are notable in magnitude. White males with A1- have an average continuation rate of 59.3, compared to white males with $\mathrm{A} 1+$, who have an average continuation rate of 44.4, a substantial difference. Black males with A1- have an average continuation rate of 51.5 , compared to black males with $\mathrm{A} 1+$, who have an average continuation rate of 34.7 , a difference also of considerable magnitude. Within each race, these differences are statistically significant, although no such differences are observed for the white or black females. Thus, DRD2 TaqI is associated with continuation to the tertiary level, but only among males (consistent with Hypotheses 1 and 5). Because of this lack of association among the white and black females, the combinatorial analyses that examine the moderating influences of social capital will be presented for white and black males only. ${ }^{3}$

\section{Mentoring, School Continuation, and DRD2: White Males}

The conceptual model suggests that mentoring will compensate for the association between DRD2 and school continuation. To address this question, we compared the continuation rates of each configuration of sets with the 47 other configurations of sets. The 'positive stand-out' and 'negative stand-out' configurations - those having statistically better and statistically worse continuation rates than the rest of the configurations - are presented in Table 2 for white males. The top panel shows the negative standouts, the bottom panel shows the positive stand-outs. For example, youth who belong to the set $\mathrm{G} \bullet \mathrm{m} \bullet \bullet \cdot \bullet \bullet \mathrm{p}$, 'A1+, no mentor, no teacher mentor, low parental socioeconomic status, not physically attractive, and personality not attractive,' have an average continuation rate of .338 versus an average continuation rate of .547 for the remaining configurations of sets, a difference that is large in magnitude and statistically significant $(F=27.52$ with 636 degrees of freedom, $p=.000)$. In contrast, youth in $\mathrm{g} \bullet \mathrm{M} \bullet \mathrm{T} \bullet \mathrm{S} \bullet \mathrm{A} \bullet \mathrm{P}$ - belonging to the 'opposite' set have an average continuation rate of .792 . and this difference is also statistically significant $(F=35.23$ with 636 degrees of freedom, $p=.000$ ). Indeed, these two configurations have the lowest and highest probabilities of continuation, respectively.

Using Boolean logic, the stand-out configurations shown in Table 2 reduce to three parsimonious solutions: White males, positive stand-out: $\mathrm{g} \cdot \mathrm{M} \cdot \mathrm{S}$ or $\mathrm{g} \cdot \mathrm{M} \cdot \mathrm{T} \cdot \mathrm{s} \cdot \mathrm{A} \cdot \mathrm{P}$

White males, negative stand-outs: $\mathrm{m} \cdot \mathrm{s}$ or $\mathrm{G} \cdot \mathrm{t} \cdot \mathrm{s}$

That is, two configurations produce better-thanaverage continuation rates: A1- coupled with a 
Table 2

Continuation Rates for Stand-Out Configurations of Sets: White Males

\begin{tabular}{|c|c|c|c|c|}
\hline Configuration of sets & Continuation rate & $\begin{array}{c}\text { Continuation for } \\
\text { other configurations }\end{array}$ & $F, p$ & $\begin{array}{c}\text { Best fit } \\
n\end{array}$ \\
\hline $\mathrm{G} \cdot \mathrm{m} \bullet \bullet \cdot s \bullet a \bullet p$ & 0.338 & 0.547 & $27.52, .000$ & 44.9 \\
\hline$G \bullet M \bullet t \bullet s \bullet a \bullet p$ & 0.346 & 0.545 & $22.94, .000$ & 37.6 \\
\hline$G \bullet m \bullet t \bullet s \bullet A \bullet P$ & 0.368 & 0.541 & $14.75, .000$ & 23.9 \\
\hline$G \bullet m \bullet \bullet \bullet \bullet A \bullet p$ & 0.384 & 0.537 & $12.78, .000$ & 6.9 \\
\hline$G \bullet M \bullet t \bullet s \bullet a \bullet P$ & 0.39 & 0.54 & $13.49, .000$ & 13.5 \\
\hline$G \bullet M \bullet \bullet \bullet s \bullet A \bullet p$ & 0.398 & 0.538 & $11.29, .001$ & 5.0 \\
\hline$g \bullet m \bullet t \bullet \bullet \bullet \bullet p$ & 0.399 & 0.545 & $13.96, .000$ & 50.5 \\
\hline$G \bullet m \bullet \bullet \bullet s \bullet a \bullet P$ & 0.407 & 0.538 & $8.77, .003$ & 5.6 \\
\hline$G \bullet M \bullet t \bullet s \bullet A \bullet P$ & 0.41 & 0.541 & $8.91, .003$ & 27.4 \\
\hline$g \bullet m \bullet t \bullet \bullet \cdot P$ & 0.435 & 0.539 & $8.15, .004$ & 8.4 \\
\hline$G \bullet M \bullet t \bullet S \bullet a \bullet p$ & 0.441 & 0.538 & $4.09, .044$ & 29.0 \\
\hline$g \bullet m \bullet t \bullet s \bullet A \bullet p$ & 0.457 & 0.538 & $5.21, .023$ & 7.8 \\
\hline$g \bullet m \bullet t \bullet s \bullet A \bullet P$ & 0.465 & 0.539 & $3.89, .049$ & 21.7 \\
\hline$g \bullet M \bullet t \bullet S \bullet a \bullet p$ & 0.622 & 0.533 & $5.91, .015$ & 34.4 \\
\hline$g \bullet M \bullet \bullet \bullet S \bullet a \bullet P$ & 0.634 & 0.535 & $7.93, .005$ & 15.7 \\
\hline$g \bullet M \bullet \bullet \bullet S \bullet A \bullet p$ & 0.635 & 0.535 & $7.61, .006$ & 14.1 \\
\hline$g \bullet M \bullet T \bullet s \bullet a \bullet P$ & 0.657 & 0.537 & $4.85, .028$ & 2.4 \\
\hline$g \bullet M \bullet T \bullet s \bullet A \bullet P$ & 0.684 & 0.536 & $7.30, .007$ & 10.9 \\
\hline$g \bullet M \bullet \bullet \bullet S \bullet A \bullet P$ & 0.69 & 0.526 & $21.36, .000$ & 50.7 \\
\hline$g \bullet M \bullet T \bullet S \bullet A \bullet p$ & 0.716 & 0.537 & $12.08, .001$ & 7.0 \\
\hline$g \bullet M \bullet T \bullet S \bullet a \bullet p$ & 0.729 & 0.531 & $16.82, .000$ & 25.5 \\
\hline$g \bullet M \bullet T \bullet S \bullet a \bullet P$ & 0.752 & 0.536 & $24.06, .000$ & 3.9 \\
\hline$g \bullet M \bullet T \bullet S \bullet A \bullet P$ & 0.792 & 0.531 & $35.23, .000$ & 25.3 \\
\hline
\end{tabular}

Note: Significance level for $F$ test with 636 degrees of freedom.

mentor and high socioeconomic parents or A1coupled with a mentor and low socioeconomic parents and all of the following: a teacher mentor, physical attractiveness, and an attractive personality. Thus, having A1- is a necessary component of positive stand-out continuation rates. Further, in the case of high socioeconomic parents, any mentor will suffice, be he/she a teacher, friend, minister/rabbi, and so forth. This pattern is consistent with the idea that high socioeconomic status students attract high socioeconomic status informal mentors. In contrast, in the case of low socioeconomic parents, the mentor must be a teacher and the student must be attractive physically and in personality. Perhaps low socioeconomic status students must attract teachers as bridging capital and they do so through their personal attractiveness.

The negative stand-outs all involve low socioeconomic status. Additionally, white males lacking a mentor do less well, as do males with $\mathrm{A} 1+$ and low socioeconomic status and no teacher mentor. Taken as a whole, the results suggest the importance of socioeconomic status (or, in the alternative, attractiveness), A1+, and mentors, especially teachers for low socioeconomic status students. Follow-up analyses reveal that mentoring matters less for students with $\mathrm{A} 1+$ : there is a 14.4 percentage point increase in continuation rates for students with A1- (i.e., comparing $\mathrm{g} \bullet \mathrm{m}$ with $\mathrm{g} \bullet \mathrm{M}, .504$ versus .648, respectively), in contrast to 4.4 percentage point increase for youth with $\mathrm{A} 1+($ i.e., comparing $\mathrm{G} \bullet \mathrm{m}$ with $\mathrm{G} \bullet \mathrm{M}, .431$ versus .475 , respectively).

Table 3 shows the continuation rates within the configurations of sets for A1+ and A1- white males. The table is divided into three panels. The top panel shows youth with no genetic differences in average continuation rates. Common to every configuration in this panel is $\mathrm{m} \bullet \mathrm{t}$ - no mentor (and thus no teacher mentor). In contrast, the bottom panel shows youth with no genetic difference in continuation rates. Common to every configuration in this panel is $\mathrm{M} \bullet \mathrm{T}$, having a teacher as mentor. Finally, the middle panel shows configurations with higher continuation rates among those students with A1- compared to students with A1+. Common to all of these configurations is $\mathrm{M} \bullet t$, having a mentor who is not a teacher. That all 24 configurations would fall perfectly into these three groups provides striking evidence to the effect that, among white males, (1) nonteacher mentors benefit students with A1-; and (2) only teachers can compensate for DRD2 A1+, and they do so completely. 
Table 3

Continuation Rates by DRD2 Allele Within Configurations: White Males

\begin{tabular}{|c|c|c|c|}
\hline Configuration of sets & $\begin{array}{l}\text { Continuation rate } \\
\text { A1- }\end{array}$ & $\begin{array}{l}\text { Continuation rate } \\
\qquad \mathrm{A} 1+\end{array}$ & $F, p$ \\
\hline$m \bullet t \bullet s \bullet a \bullet p$ & 0.399 & 0.338 & $1.07, .300$ \\
\hline$m \bullet t \bullet s \bullet a \bullet P$ & 0.435 & 0.407 & $0.2, .655$ \\
\hline$m \bullet t \bullet s \bullet A \bullet p$ & 0.457 & 0.384 & $1.43, .233$ \\
\hline$m \bullet t \bullet s \bullet A \bullet P$ & 0.465 & 0.368 & $2.37, .124$ \\
\hline$m \bullet t \bullet S \bullet a \bullet p$ & 0.536 & 0.49 & $0.51, .476$ \\
\hline$m \bullet t \bullet S \bullet a \bullet P$ & 0.542 & 0.486 & $0.72, .397$ \\
\hline$m \bullet t \bullet S \bullet A \bullet p$ & 0.519 & 0.459 & $0.85, .356$ \\
\hline$m \bullet t \bullet S \bullet A \bullet P$ & 0.597 & 0.51 & $1.76, .185$ \\
\hline 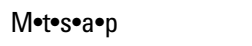 & 0.518 & 0.346 & $7.71, .006$ \\
\hline$M \bullet t \bullet s \bullet a \bullet P$ & 0.545 & 0.39 & $6.48, .011$ \\
\hline$M \bullet t \bullet s \bullet A \bullet p$ & 0.592 & 0.398 & $10.22, .001$ \\
\hline$M \bullet t \bullet s \bullet A \bullet P$ & 0.609 & 0.41 & $10.16, .002$ \\
\hline$M \bullet t \bullet S \bullet a \bullet p$ & 0.622 & 0.441 & $7.99, .005$ \\
\hline$M \bullet t \bullet S \bullet a \bullet P$ & 0.634 & 0.465 & $7.72, .006$ \\
\hline$M \bullet t \bullet S \bullet A \bullet p$ & 0.635 & 0.48 & $6.2, .013$ \\
\hline$M \bullet t \bullet S \bullet A \bullet P$ & 0.69 & 0.524 & $7.55, .006$ \\
\hline$M \bullet T \cdot s \bullet a \bullet p$ & 0.54 & 0.587 & $0.21, .644$ \\
\hline$M \bullet T \bullet s \bullet a \bullet P$ & 0.657 & 0.596 & $0.36, .549$ \\
\hline$M \bullet T \bullet s \bullet A \bullet p$ & 0.625 & 0.64 & $0.02, .877$ \\
\hline$M \bullet T \bullet S \bullet A \bullet P$ & 0.684 & 0.643 & $0.17, .676$ \\
\hline$M \bullet T \bullet S \bullet a \bullet p$ & 0.729 & 0.627 & $1.19, .275$ \\
\hline$M \bullet T \bullet S \bullet a \bullet P$ & 0.752 & 0.637 & $1.62, .203$ \\
\hline$M \bullet T \bullet S \bullet A \bullet p$ & 0.716 & 0.663 & $0.31, .578$ \\
\hline$M \bullet T \bullet S \bullet A \bullet P$ & 0.792 & 0.668 & $1.88, .171$ \\
\hline
\end{tabular}

Note: Significance level for $F$ test with 636 degrees of freedom.

Figure 1 summarizes the broad patterns of Table 3. For white males, those youth without a mentor have lowest continuation rates irrespective of DRD2 allele. For youth with an informal mentor who is not a teacher, only students with A1- benefit and the difference in average continuation rates between risk groups is appreciable in magnitude and statistically significant (as documented in Table 3, middle panel). Finally, teachers as mentors fully compensate for A1+: $70 \%$ of A1- students continue to college, while $65 \%$ of $\mathrm{A} 1+$ risk students continue, and this difference is statistically insignificant.

Nevertheless, what percentage of white male students have $\mathrm{A} 1+$ and attract a mentor, especially a teacher as mentor? To address this question, we crosstabulated DRD2 allelic variants with five mentor categories (none, friend, relative, teacher, and community). The omnibus chi-squared test is marginally significant, indicating that DRD2 risk status is weakly associated with the distribution of these mentorship statuses (chi-squared with 4 degrees of freedom $=7.99, p=.092)$. An inspection of the table nonetheless suggested a significant difference involving teacher mentors and an additional test revealed a statistically significant difference: DRD2 A1+ is asso- ciated with fewer teacher mentors $(13.10 \%)$ than A1(18.72; chi-squared with 1 degree of freedom $=4.84$, $p=.028)$. Indeed, 46 white males have A1+ and a teacher as mentor.

Consistent with expectations, then, teachers fully compensate for DRD2 A1+ with respect to educational continuation beyond secondary school, but youth with $\mathrm{A} 1+$ are less likely to attract teachers as mentors in the first place.

\section{Mentoring, School Continuation, and DRD2: Black Males}

Turning to black males, we once again begin by examining positive and negative stand-out configurations. The top panel of Table 4 shows negative stand-outs, those configurations with lower than average continuation rates, and the bottom panel shows the positive stand-outs. Parsimonious solutions can be obtained by applying Boolean logic to the table, which results in: Black males, positive stand-out: $\mathrm{g} \cdot \mathrm{M} \cdot \mathrm{t} \cdot \mathrm{S}$ or $\mathrm{g} \cdot \mathrm{M} \cdot \mathrm{t} \cdot \mathrm{s} \cdot \mathrm{A} \cdot \mathrm{P}$ $\mathrm{G} \cdot \mathrm{M} \cdot \mathrm{T} \cdot \mathrm{S}$ or $\mathrm{G} \cdot \mathrm{M} \cdot \mathrm{T} \cdot \mathrm{s} \cdot \mathrm{A} \cdot \mathrm{P}$

Black males, negative stand-outs: $\mathrm{G} \cdot \mathrm{m} \cdot \mathrm{s} \cdot \mathrm{a}$ or $\mathrm{G} \cdot \mathrm{M} \cdot \mathrm{t} \cdot \mathrm{s} \cdot \mathrm{p}$

That is, black males do better than average in several circumstances. First, among black males with A1-, a 


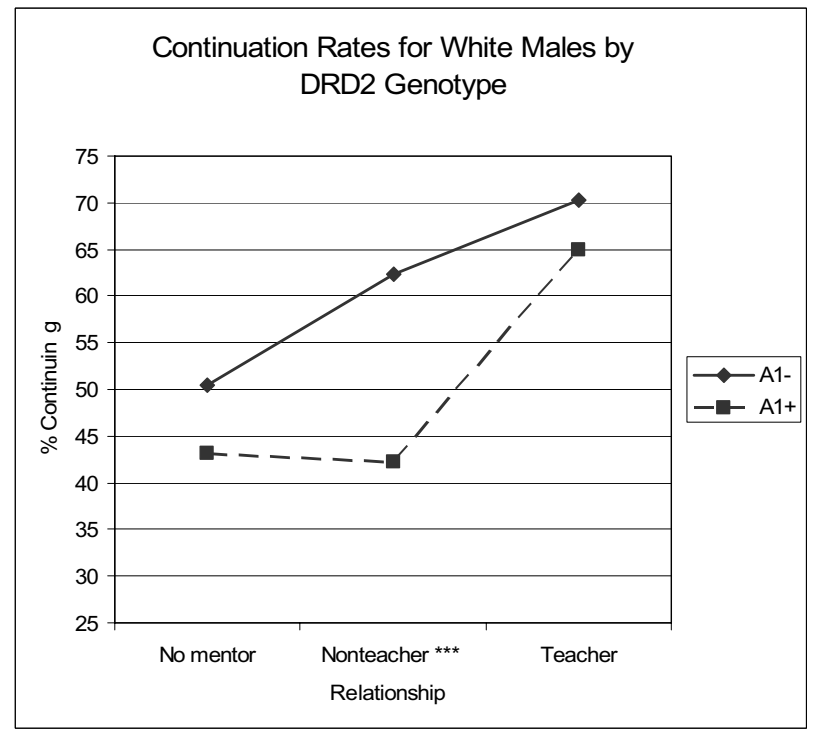

Figure 1

Per cent white males continuing their education past secondary school by DRD2 allelic variant and mentorship statuses (Add Health).

nonteacher mentor and high socioeconomic status or both physical and personal attractiveness. Second, among black males with $\mathrm{A} 1+$, a teacher mentor and high socioeconomic status or both physical and personal attractiveness. These results are especially noteworthy in two respects. As was observed among white males, physical and personality attractivess can substitute for parental high socioeconomic status. Black males with $\mathrm{A} 1+$ also require a teacher as a mentor. However, white males with A1- require a teacher mentor, while black males require a nonteacher mentor.

Common to the negative stand-outs for black males are $\mathrm{A} 1+$ and low socioeconomic status. These factors may be coupled with no mentor and low physical attractiveness or a nonteacher mentor and low attractiveness of personality. Common to white and black males with lower than average continuation rates, then, is no mentor and low socioeconomic status or DRD2 A1+ and no teacher mentor. Once again, the results highlight the importance of socioeconomic status, $\mathrm{A} 1+$, and mentors, although there is a high level of contingency operating among these factors.

Table 5 shows the average continuation rates within configurations of sets and across groups defined by DRD2 allelic variant. Once again, the table is divided into three panels. The top panel shows configurations including $m \bullet t$ type of mentorship. These configurations generally have low continuation rates and there are no differences by DRD2 allele. This pattern was also observed for white males. Configurations in the middle panel differ significantly, with black males with A1- having significantly higher average continuation rates.
Common to all of these cases is $M \bullet t$, indicating the presence of a mentor who is not a teacher. This pattern was also observed among the white males. Finally, the bottom panel $(\mathrm{M} \cdot \mathrm{T})$ shows configurations that are, with few exceptions, significantly different in their continuation rates, with black males with A1having the lowest average continuation rates.

The patterns suggested by Table 5 are shown in Figure 2. For black males without mentors, DRD2 status does not make a difference for average continuation rates (although the magnitude of the difference may appear large, the difference is statistically insignificant). Black males benefit from a mentor who is not a teacher only if they have A1-. These patterns were both observed for white males. Likewise, only teachers as mentors compensate for A1+. Unlike white makes, however, black males with A1- have much lower average contuinuation rates. While these data points are based on few cases (12 for A1+ and 19 for A1-), the difference is statistically significant. We return to this finding in the final section of the paper.

The results show that, once again, the negative association between DRD2 status and average continuation rate is fully attenuated only by mentors who are teachers. A cross-tabulation of DRD2 status by types of mentors yields an insignificant omnibus test (chi-squared with 4 degrees of freedom $=4.69$, $p=.321$ ), indicating no global differences in DRD2 status and mentoring. However, an inspection of the table reveals that black males with $\mathrm{A} 1+$ are less likely to have a teacher for a mentor $(9.84 \%$ versus $19.03 \%$, respectively), which was confirmed by a post hoc test (chi-squared with 1 degree of freedom $=3.94$, $p=.047)$. In other words, teachers as mentors are uniquely capable of eliminating DRD2 differences in

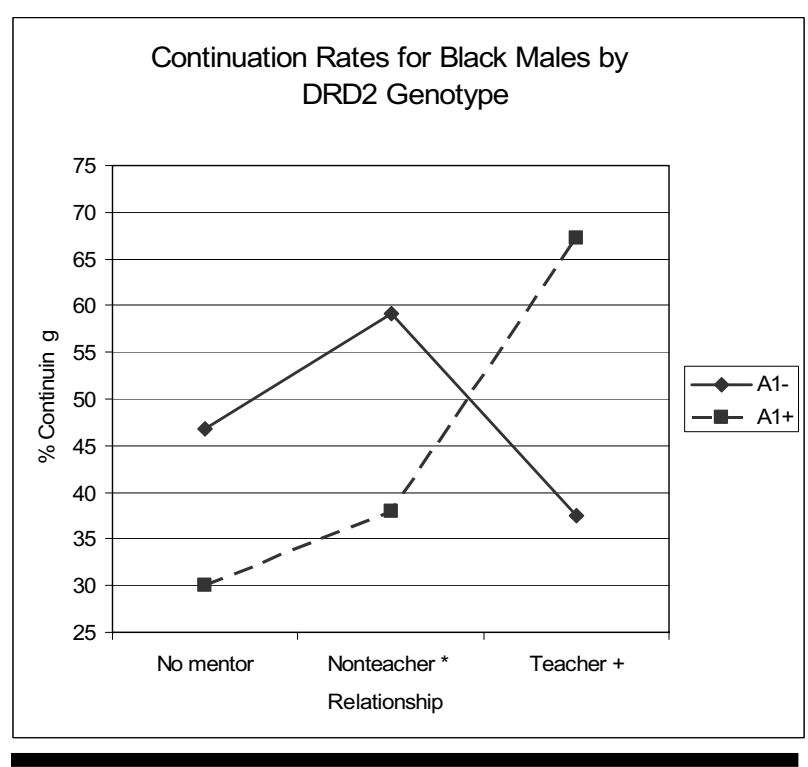

Figure 2

Per cent black males continuing their education past secondary school by DRD2 allelic variant and mentorship statuses (Add Health). 
Table 4

Continuation Rates for Stand-Out Configurations of Sets: Black Males

\begin{tabular}{|c|c|c|c|c|}
\hline Configuration of sets & Continuation rate & $\begin{array}{c}\text { Continuation for } \\
\text { other confiigurations }\end{array}$ & $F, p$ & $\begin{array}{c}\text { Best fit } \\
n\end{array}$ \\
\hline$G \bullet m \bullet t \bullet s \bullet a \bullet p$ & 0.256 & 0.443 & $5.93, .016$ & 16.7 \\
\hline$G \bullet m \bullet t \bullet s \bullet a \bullet P$ & 0.274 & 0.439 & $6.19, .014$ & 3.0 \\
\hline$G \bullet M \bullet \bullet \bullet s \bullet a \bullet p$ & 0.282 & 0.449 & $7.63, .006$ & 20.4 \\
\hline$G \bullet M \bullet t \bullet s \bullet A \bullet p$ & 0.287 & 0.438 & $5.99, .015$ & 8.2 \\
\hline$g \bullet M \bullet t \bullet S \bullet a \bullet p$ & 0.618 & 0.435 & $6.75, .010$ & 2.4 \\
\hline$g \bullet M \bullet t \bullet s \bullet A \bullet P$ & 0.629 & 0.432 & $6.26, .013$ & 11.3 \\
\hline$g \bullet M \bullet t \bullet S \bullet a \bullet P$ & 0.642 & 0.436 & $7.59, .006$ & 1.9 \\
\hline$g \bullet M \bullet t \bullet S \bullet A \bullet p$ & 0.667 & 0.433 & $11.82, .001$ & 3.0 \\
\hline$g \bullet M \bullet t \bullet S \bullet A \bullet P$ & 0.726 & 0.43 & $21.15, .000$ & 6.7 \\
\hline$G \bullet M \bullet T \bullet S \bullet A \bullet P$ & 0.772 & 0.434 & $8.06, .005$ & 2.2 \\
\hline$G \bullet M \bullet T \cdot S \bullet a \bullet P$ & 0.788 & 0.435 & $5.13, .025$ & 1.8 \\
\hline$G \bullet M \bullet T \bullet S \bullet a \bullet p$ & 0.809 & 0.434 & $10.11, .002$ & 1.2 \\
\hline$G \bullet M \bullet T \bullet S \bullet A \bullet p$ & 0.825 & 0.436 & $11.6, .001$ & 0 \\
\hline$G \bullet M \bullet T \bullet S \bullet A \bullet P$ & 0.896 & 0.434 & $30.84, .000$ & 1.9 \\
\hline
\end{tabular}

Note: Significance level for $F$ test with 636 degrees of freedom.

average continuation rates for black males, but $\mathrm{A} 1+$ is associated with a lowered likelihood of having a teacher as a mentor.

\section{Conclusions and Discussion}

Although helping relationships are central to positive development and surmounting challenges in life, few studies appreciate that receiving help is endogenous and that its effectiveness depends on the nature of the proffered help. With respect to endogeneity, helpers are not randomly distributed across people: some types of people are more likely to elicit and maintain a relationship with a helper. If people in need of help are defined in terms of genetic propensity for risky behavior, this pattern of endogeniety suggests a gene-environment correlation according to which such people are least likely to be party to a helping relationship. With respect to effectiveness, some helpers are uniquely suited to help those in need because of their special skills and knowledge. The well-intentioned neighbor or friend may or may not be capable of helping with specific problems. Once again, if need is defined by genetic propensity for risky behaviors, this reality suggests a gene-environment interaction according to which a specific risk's negative implications are attenuated by certain types of help but not others. In short, perhaps people in need of specific help are likely to benefit from it but also unlikely to receive it. Cast in terms of DRD2, perhaps students with A1+ will benefit from teachers as mentors but be less likely to receive such support than students with A1-.

These complexities have been illustrated with the strategic example of DRD2, mentors, and continuation beyond secondary school. After acknowledging limitations to our empirical example, we discuss the findings and then briefly consider the value of combinatoric approaches to the study of genetics, social context, and the life course.

First, there is no 'college gene.' Success in school and the likelihood of extending one's education beyond secondary school undoubtedly depend on many skills that are influenced by genetic action. Our theoretical model posits that risk associated with DRD2 increases the likelihood of behaviors that undermine the student's ability to do well in school and thereby the likelihood of continuing one's education beyond high school. But what are these behaviors that trace to DRD2 and that interfere with school performance? Prior research is suggestive in only molar terms: impulsivity, poor ability to learn based on rewards and punishments, poor ability to delay gratification. Exactly how students differ in their school-related behavioral repertoires is unknown, however.

In specific terms, how are DRD2 A1+ students different from A1- students? This question assumes special importance given that the continuation rates of both white and black girls did not differ by DRD2 allelic variant, and given the DRD2-teacher as mentor correlation. Males with DRD2 A1+ must differ phenotypically from males with A1- and, in turn, the A1+ males differ from the A1+ females. But how? The question calls for a genetically informed, classroombased ethnography. A related question for future research posed by the findings concerns black males and teachers as mentors. Drawing on the work of Stanton-Salazar and Dornbusch (1995), we hypothesized that black males would not benefit from mentors who are 'institutional agents' of the school because such mentors typically do not share 'subcultural values and norms' with racial and ethnic minority 
Table 5

Continuation Rates by DRD2 Allele Within Configurations: Black Males

\begin{tabular}{|c|c|c|c|}
\hline Configuration of sets & $\begin{array}{c}\text { Continuation rate } \\
\qquad \mathrm{A} 1-\end{array}$ & $\begin{array}{c}\text { Continuation rate } \\
\qquad \mathrm{A} 1+\end{array}$ & $F, p$ \\
\hline$m \bullet t \bullet s \bullet a \bullet p$ & 0.415 & 0.256 & $1.83, .177$ \\
\hline$m \bullet t \bullet s \bullet a \bullet P$ & 0.471 & 0.274 & $2.89, .090$ \\
\hline$m \bullet \bullet \bullet s \bullet A \bullet p$ & 0.432 & 0.319 & $0.91, .342$ \\
\hline$m \bullet t \bullet s \bullet A \bullet P$ & 0.423 & 0.298 & $1.09, .299$ \\
\hline$m \bullet t \bullet S \bullet a \bullet p$ & 0.507 & 0.354 & $1.59, .209$ \\
\hline$m \bullet t \cdot S \bullet a \bullet P$ & 0.495 & 0.357 & $1.3, .255$ \\
\hline$m \bullet \bullet \bullet S \bullet A \bullet p$ & 0.464 & 0.363 & $0.67, .415$ \\
\hline$m \bullet t \bullet S \bullet A \bullet P$ & 0.533 & 0.412 & $0.91, .342$ \\
\hline$M \bullet \bullet \bullet s \bullet a \bullet p$ & 0.529 & 0.282 & $6.06, .015$ \\
\hline$M \bullet t \bullet s \bullet a \bullet P$ & 0.579 & 0.351 & $4.5, .035$ \\
\hline$M \bullet \bullet \bullet s \bullet A \bullet p$ & 0.559 & 0.287 & $6.88, .009$ \\
\hline$M \bullet \bullet \bullet s \bullet A \bullet P$ & 0.629 & 0.387 & $5, .026$ \\
\hline$M \bullet \bullet \bullet S \bullet a \bullet p$ & 0.618 & 0.428 & $3.04, .083$ \\
\hline$M \bullet \bullet \cdot S \bullet a \bullet P$ & 0.642 & 0.381 & $6.32, .013$ \\
\hline$M \bullet \bullet \bullet S \bullet A \bullet p$ & 0.667 & 0.32 & $11.71, .001$ \\
\hline$M \bullet \bullet \bullet S \bullet A \bullet P$ & 0.726 & 0.428 & $8.75, .003$ \\
\hline$M \bullet T \bullet s \bullet a \bullet p$ & 0.33 & 0.479 & $0.58, .449$ \\
\hline$M \bullet T \bullet s \bullet a \bullet P$ & 0.353 & 0.612 & $1.69, .196$ \\
\hline$M \bullet T \bullet s \bullet A \bullet p$ & 0.281 & 0.673 & $4.71, .031$ \\
\hline$M \bullet T \bullet S \bullet A \bullet P$ & 0.356 & 0.772 & $6.11, .014$ \\
\hline$M \bullet T \cdot S \bullet a \bullet p$ & 0.397 & 0.809 & $4.91, .028$ \\
\hline$M \cdot T \cdot S \cdot a \cdot P$ & 0.39 & 0.788 & $4.08, .045$ \\
\hline$M \bullet T \cdot S \bullet A \bullet p$ & 0.361 & 0.825 & $6.34, .013$ \\
\hline$M \bullet T \cdot S \cdot A \cdot P$ & 0.375 & 0.896 & $11.79, .001$ \\
\hline
\end{tabular}

Note: Significance level for $F$ test with 636 degrees of freedom.

students (p. 119). This expectation proved insightful with respect to black males with A1-, but not for black males with DRD2 A1+. There is very little research on minorities and school-based mentors and the finding for the latter group is presently inexplicable. Again, the new questions raised seem best suited to a genetically informed ethnography.

Second, given limitations in sample size and measures, we can only consider a small number of relevant indicators of mentors and genetic candidates. As with all studies conducted in a qualitative comparative framework, our models were formulated with attention to theory and prior research. Nevertheless, there are numerous genetic and social candidates that could plausibly be incorporated into our models. With the larger genetic sample of Wave IV of Add Health, we will be able to examine many additional, plausible possibilities.

Third, because of our reliance on quasi-experimental methods, we can not rule out the possibility of spurious findings. Perhaps DRD2 is not in itself important but rather it is closely associated with other genetic candidates that are causally linked to behaviors that detract from one's educational accomplishments. Our conceptual model is based on persuasive evidence with respect to genetic and social mechanisms and indeed it generated a set of hypotheses that proved insightful. Nevertheless, like all quasi-experimental studies, the present findings support provisional conclusions that call for further study informed by other theoretical frameworks and tested with other samples, measures, designs, and statistical models.

Finally, QCA is attractive as a descriptive framework capable of detecting high levels of contingency among independent variables, but this is accomplished with a multitude of contrasts, raising the possibility of false positives. Replication is especially important in these circumstances. Indeed, our findings comport closely with our expectations, which provides a solid justification for future replications.

\section{Mentor-DRD2 Interplay}

The results suggest that DRD2 A1+ is associated with decreased likelihood of school continuation (consistent with Hypothesis 1), but only for boys (consistent with Hypothesis 4) and these trends are considerable in magnitude. Further, as expected, teachers as mentors are able to compensate for this risk completely for white and black males and these trends are also considerable 
in magnitude (Hypothesis 2). Among white males with A $1+$, about $43 \%$ with no mentor or a nonteacher mentor continued their educations beyond high school, compared to $60 \%$ with a teacher as mentor (see Figure 1), a pattern yet more pronounced among black males (see Figure 2). In contrast, nonteacher mentors had no effect on average continuation rates, consistent with Hypothesis 3. Perhaps because of their knowledge of the educational system and understanding of the student, teachers are uniquely qualified to advise students about their educations, including addressing issues of behavioral comportment in the classroom. It may be that, lacking this unique perspective, nonteacher mentors were ineffective in fostering educational continuation.

Nevertheless, white and black males with A1+ were significanty less likely to report having a teacher as mentor, consistent with Hypothesis 4 and indicating a gene-environment correlation. Conceptual considerations suggested that teachers were less likely to mentor students with A1+ than students without such risk perhaps because the former's consistent patterns of impulsive behaviors and poor self-regulation made them 'poor investments' of limited helping resources.

Finally, Hypothesis 6 predicted that the positive effects of teachers as mentors would not be observed for black males. The evidence on this point is mixed. Black males with A1+ benefit substantially from teachers as mentors and, in this sense, the (null) hypothesis is disconfirmed. Black males with A1show the hypothesized pattern, however. As suggested, an ethnographic study might help clarify the meaning of this pattern.

The over-arching pattern suggested by these data for the white and black males is clear: the link between DRD2 A1+ and continuation beyond high school is fully attenuated by mentors who are teachers, but males with $\mathrm{A} 1+$ are less likely to have a mentor who is a teacher then males with A1-. As with all studies documenting gene-environment interplay, replication is essential.

\section{Combinatoric Strategies for Studying G-E Interplay}

In addition to revealing new findings and raising new questions about the role of mentors in the educational process, the present analyses also illustrate the usefulness of a combinatoric approach to the study of gene-environment interplay. Combinatoric approaches begin with the assumption that people belong to sets and that sets form configurations. The sets may be defined by genetic, social, psychological, and other types of variables. The immediate issues then become: which configurations of sets exist? How common is membership in each configuration of sets? And how is membership in a configuration associated with other variables, both antecedents and consequences?

As shown in the present example, such an approach is well-suited to studying gene-environment correlations and interactions simultaneously. What allele-social context configurations exist? And what is the likelihood of being a member of a specific configuration? These are questions that get to the heart of gene-environment correlations. In the present case, DRD2 A1+ decreased the likelihood of belonging to any configuration that included a teacher as mentor. Aside from the percentage of people populating each configuration, are combinations of specific alleles and specific contextual behaviors related to a criterion variable in a nonlinear fashion? This is a question about gene-environment interactions. In the present case, mentors who were not teachers increased the probability of continuation, but only for students with A1-. Teachers alone, acting as mentors, were capable of neutralizing DRD2 A1+.

Beyond the specific example of DRD2, mentoring, and education, combinatoric strategies are a presently neglected but valuable analytic approach to the study of genetics and social context. Given specific genotypes, what social circumstances are people in? And how are these patterns, in turn, related to health and well-being? Sociological and psychological findings clearly establish the importance of 'significant others' for positive development. Future research will ideally join insights from behavioral genetics with these findings, drawing on analytic strategies that are sensitive to ongoing gene-environment correlations and interactions.

\section{Acknowledgments}

This research uses data from the Add Health Study designed by J. Richard Udry, Peter S. Bearman, and Kathleen Mullan Harris and the Add Health Wave IV Program Project directed by Kathleen Mullan Harris (Grant 3P01 HD031921), funded by the National Institute of Child Health and Human Development with cooperative funding from 17 other agencies. We gratefully acknowledge support from NICHD to Glen H. Elder, Jr. and Michael J. Shanahan through their subproject to the Add Health Wave IV Program Project (Grant 3P01 HD031921). We thank Kyle Longest for programming assistance and advice, and Jennifer Harris and an anonymous reviewer for insightful comments.

The authors acknowledge funding support from the National Institute on Aging. The views expressed are those of the authors of this paper, and not the National Institute on Aging, the National Institutes of Health or the Department of Health and Human Services.

\section{Endnotes}

1 Membership in a set is indicated by an upper case letter; lack of membership is indicated by a lower case letter, the Boolean 'and' is indicated by the symbol $\bullet$, and the Boolean 'or' is indicated by the symbol + . 
2 These ratios and their standard errors are computed taking family clustering into account by using the command-ratio, cluster()- in Stata 9.

3 Additional analyses showed that there were no configurations of social capital within which the continuation rates of risk and nonrisk females differed (either white or black).

\section{References}

Avison, W. R. (2005). Education, social status, and health. American Journal of Sociology, 110, 1511-1513.

Bourdieu, P., \& Passeron, J. C. (1990). Reproduction in education, society and culture (theory, culture and society series) (2nd ed.). Thousand Oaks, CA: Sage Publication.

Bryant, A. L., \& Zimmerman, M. A. (2003). Role models and psychosocial outcomes among African American adolescents. Journal of Adolescent Research, 18, 36-67.

Dohrenwend, B. P., Levav, I., Shrout, P. E., Schwartz, S., Naveh, G., Link, B. G., Skodol, A. E., \& Stueve, A. (1992). Socioeconomic-status and psychiatric-disorders: The causation-selection issue. Science, 255, 946-952.

Eubanks, J. H., Djabali, M., Selleri, L., Grandy, D. K., Civelli, O., McElligott, D. L., \& Evans, G. A. (1992). Structure and linkage of the D2 dopamine receptor and neural cell adhesion molecule genes on human chromosome 11q23. Genomics, 14, 1010-1018.

Geweke, J., \& Keane, M. (2000). An empirical analysis of earnings dynamics among men in the PSID: 1968-1989. Journal of Econometrics, 96, 293-356.

Glickstein, S. B., Desteno, D. A., Hof, P. R., \& Schmauss, C. (2005). Mice lacking dopamine D2 and D3 receptors exhibit differential activation of prefrontal cortical neurons during tasks requiring attention. Cerebral Cortex, 15, 1016-1024.

Grandy, D. K., Litt, M., Allen, L., Bunzow, J. R., Marchionni, M., Makam, H., Reed, L., Magenis, R. E., \& Civelli, O. (1989). The human dopamine D2 receptor gene is located on chromosome 11 at q22-q23 and identifies a TaqI RFLP. American Journal of Human Genetics, 45, 778-785.

Haberstick, B. C., \& Smolen, A. (2004). Genotyping of three single nucleotide polymorphisms following whole genome preamplification of DNA collected from buccal cells. Behavior Genetics, 34, 541-547.

Hamilton, S. F., \& Darling, N. (1996). Mentors in adolescents' lives. In K. Hurrelmann \& S. F. Hamilton (Eds.), Social problems and social contexts in adolescence: Perspectives across boundaries (pp. 199-215). New York: Aldine De Gruyter.

Jonsson, E. G., Nothen, M. M., Grunhage, F., Farde, L., Nakashima, Y., Propping, P., \& Sedvall, G. C. (1999). Polymorphisms in the dopamine D2 receptor gene and their relationships to striatal dopamine receptor density of healthy volunteers. Molecular Psychiatry, 4, 290-296.

Kidd, K. K., Pakstis, A. J., Castiglione, C. M., Kidd, J. R., Speed, W. C., Goldman, D., Knowler, W. C., Lu, R. B., \& Bonne-Tamir, B. (1996). DRD2 haplotypes containing the TaqI A1 allele: Implications for alcoholism research. Alcoholism, Clinical and Experimental Research, 20, 697-705.

Klaw, E. L., Rhodes, J. E., \& Fitzgerald, L. F. (2003). Natural mentors in the lives of African American adolescent mothers: Tracking relationships over time. Journal of Youth and Adolescence, 32, 223-232.

Kruzich, P. J., Mitchell, S. H., Younkin, A., \& Grandy, D. K. (2006). Dopamine D2 receptors mediate reversal learning in male C57BL/6J mice. Cognitive, Affective and Behavioral Neuroscience, 6, 86-90.

Livak, K. J. (1999). Allelic discrimination using fluorogenic probes and the 5' nuclease assay. Genetic Analysis: Biomolecular Engineering, 14, 143-149.

Miech, R. A., \& Shanahan, M. J. (2000). Socioeconomic status and depression over the life course. Journal of Health and Social Behavior, 41, 162-176.

Noble, E. P. (2003). D2 dopamine receptor gene in psychiatric and neurologic disorders and its phenotypes. American Journal of Medical Genetics. Part B, Neuropsychiatric Genetics, 116, 103-125.

Online Mendelian Inheritance in Man, OMIM (TM). Johns Hopkins University, Baltimore, MD. MIM Number: 126450. Retrieved 29 January, 2007, from http://www.ncbi.nlm.nih.gov/omim/

Pattij, T., Janssen, M. C., Vanderschuren, L. J., Schoffelmeer, A. N., \& van Gaalen, M. M. (2007). Involvement of dopamine $\mathrm{D}(1)$ and $\mathrm{D}(2)$ receptors in the nucleus accumbens core and shell in inhibitory response control. Psychopharmacology, 191, 587-598.

Pessiglione, M., Seymour, B., Flandin, G., Dolan, R. J., \& Frith, C. D. (2006). Dopamine-dependent prediction errors underpin reward-seeking behaviour in humans. Nature, 442, 1042-1045.

Pezze, M. A., Dalley, J. W., \& Robbins, T. W. (2006). Differential roles of dopamine D1 and D2 receptors in the nucleus accumbens in attentional performance on the five-choice serial reaction time task. Neuropsychopharmacology, 32, 273-283.

Oohjalainen, T., Rinne, J. O., Nagren, K., Lehikoinen, P., Anttila, K., Syvalahti, E. K., \& Hietala, J. (1998). The A1 allele of the human D2 dopamine receptor gene predicts low D2 receptor availability in healthy volunteers. Molecular Psychiatry, 3, 256-260.

Portes, A. (1998). Social capital: Its origins and applications in modern sociology. Annual Review of Sociology, 24, 1-24.

Ragin, C. C. (1989). The comparative method: Moving beyond qualitative and quantitative strategies. Berkeley, CA: University of California Press. 
Ragin, C. C. (2000). Fuzzy-set social science. Chicago, IL: University Of Chicago Press.

Restuccia, D., \& Urrutia, C. (2004). Intergenerational persistence of earnings: The role of early and college education. American Economic Review, 94, 1354-1378.

Rhodes, J. E. (2002). Stand by me: The risks and rewards of mentoring today's youth. Cambridge, MA: Harvard University Press.

Rhodes, J. E., Contreras, J. M., \& Mangelsdorf, S. C. (1994). Natural mentor relationships among latina adolescent mothers: Psychological adjustment, moderating processes, and the role of early parental acceptance. American Journal of Community Psychology, 22, 211-227.

Rhodes, J. E., Ebert, L., \& Fischer, K. (1992). Natural mentors - An overlooked resource in the social networks of young, African-American mothers. American Journal of Community Psychology, 20, 445-461.

Rhodes, J. E., Grossman, J. B., \& Resch, N. L. (2000). Agents of change: Pathways through which mentoring relationships influence adolescents' academic adjustment. Child Development, 71, 1662-1671.

Ross, C. E., \& Wu, C. L. (1995). The links between education and health. American Sociological Review, 60, 719-745.

Rubin, D. B. (2004). Multiple imputation for nonresponse in surveys. New York: Wiley-Interscience.

Schnittker, J. (2004). Education and the changing shape of the income gradient in health. Journal of Health and Social Behavior, 45, 286-305.

Sewell, W. H., Haller, A. O., \& Portes, A. (1969). Educational and early occupational attainment process. American Sociological Review, 34, 82-92.
Smith, J. W., Fetsko, L. A., Xu, R., \& Wang, Y. (2002). Dopamine D2L receptor knockout mice display deficits in positive and negative reinforcing properties of morphine and in avoidance learning. Neuroscience, $113,755-765$.

Stanton-Salazar, R. D., \& Dornbusch, S. M. (1995). Social capital and the reproduction of inequality: Information networks among Mexican-origin highschool students. Sociology of Education, 68, 116-135.

Thompson, J., Thomas, N., Singleton, A., Piggott, M., Lloyd, S., Perry, E. K., Morris, C. M., Perry, R. H., Ferrier, I. N., \& Court, J. A. (1997). D2 dopamine receptor gene (DRD2) Taq1 A polymorphism: Reduced dopamine D2 receptor binding in the human striatum associated with the A1 allele. Pharmacogenetics, 7, 479-484.

Tran, A. H., Tamura, R., Uwano, T., Kobayashi, T., Katsuki, M., \& Matsumoto, G., \& Ono, T. (2002). Altered accumbens neural response to prediction of reward associated with place in dopamine D2 receptor knockout mice. Proceedings of the National Academy of Sciences USA, 99, 8986-8991.

van Gaalen, M. M., Brueggeman, R. J., Bronius, P. F., Schoffelmeer, A. N., \& Vanderschuren, L. J. (2006). Behavioral disinhibition requires dopamine receptor activation. Psychopharmacology, 187, 73-85.

van Gaalen, M. M., van Koten, R., Schoffelmeer, A. N., \& Vanderschuren, L. J. (2006). Critical involvement of dopaminergic neurotransmission in impulsive decision making. Biological Psychiatry, 60, 66-73.

Zimmerman, M. A., \& Bingenheimer, J. B. (2002). Natural mentors and adolescent resiliency: A study with urban youth. American Journal of Community Psychology, 30, 221-243. 